\title{
Therapeutic response to thalidomide in Melkersson-Rosenthal syndrome: a case report
}

Manoel Medeiros, Jr., MD*; Maria Ilma Araujo, MD*; Newton Sales Guimarães, MD†; Luiz Antonio Rodrigues Freitas, MD $\neq$; Tania Maria Correia Silva, MD ; and Edgar Marcelino Carvalho, MD*

Background: Melkersson-Rosenthal syndrome (MRS) is a rare disorder of unknown etiology characterized by a triad of symptoms: recurrent orofacial swelling, relapsing facial palsy, and a fissured tongue. A differential diagnosis must be made with other granulomatous diseases, such as sarcoidosis and oral Crohn disease; however, the histologic findings of noncaseating, sarcoidal granulomas support the diagnosis of MRS.

Results: Many therapeutic modalities have been described for this disease. In this case report, we present a patient with MRS that was treated with thalidomide because of the identification of tumor necrosis factor $\alpha$ in the lesion by immunohistochemical analysis. This is the first reported detection of tumor necrosis factor $\alpha$ in an MRS lesion, as well the first reported use of thalidomide to treat this clinical condition.

Ann Allergy Asthma Immunol 2002;88:421-424.

\section{INTRODUCTION}

The Melkersson-Rosenthal syndrome (MRS) is a rare disorder of unknown etiology characterized by a triad of recurrent orofacial swelling, relapsing facial paralysis, and a fissured tongue. Exacerbations and recurrences are common. The orofacial swelling is characterized by fissured, reddishbrown, swollen, nonpruritic lips or firm edema of the face, whereas the facial palsy is similar to that found in Bell palsy. The fissured tongue is seen in one-third to one-half of all patients, and, although it is the least common manifestation, its presence assists in the diagnosis. ${ }^{1-3}$ In addition to these symptoms, a common presenting complain in MRS is gingival hypertrophy. ${ }^{4}$ The classic triad of symptoms is not

* Immunology Service, Hospital Universitario Prof. Edgar Santos, Federal University of Bahia, Salvador, Bahia, Brazil.

$\dagger$ Division of Dermatology, Department of Medicine, Federal University of Bahia, Salvador, Bahia, Brazil.

† Laboratory of Pathology and Cellular Biology, Oswaldo Cruz Foundation (FIOCRUZ), Salvador, Bahia, Brazil.

Received for publication June 14, 2001.

Accepted for publication in revised form November 30, 2001. always observed, making the diagnosis more difficult. However, the histopathologic findings of noncaseating, sarcoidal granulomas support the diagnosis. ${ }^{1,2}$

Different factors associated with the disease include genetic inheritance, ${ }^{5}$ intolerance to food additives, ${ }^{6}$ delayed hypersensitivity to dietary proteins ${ }^{7}$ or cow's milk proteins, ${ }^{8}$ and delayed hypersensitivity to metals, such as cobalt. ${ }^{9}$

In cases of cheilitis granulomatosa, a differential diagnosis must be made with Crohn disease and sarcoidosis. Crohn disease can be accompanied by intestinal symptoms and bone alterations, whereas in sarcoidosis, pulmonary alterations, visible on chest $\mathrm{x}$-ray, occur along with an elevation of angiotensin-converting enzyme. ${ }^{1,2}$

Many therapeutic alternatives have been suggested for the treatment of MRS, including hydroxychloroquine sulfate, ${ }^{10}$ methotrexate, ${ }^{11}$ intralesion corticosteroid injections or oral methylprednisolone, ${ }^{12}$ clofazimine, ${ }^{13}$ and surgical reduction. ${ }^{14}$ There are also reports on metronidazole ${ }^{15}$ and minocycline alone ${ }^{16}$ or associated with prednisone, ${ }^{17}$ showing that these drugs could be helpful in some cases.

In this case report, we show for the first time that tumor necrosis factor $\alpha$
(TNF- $\alpha$ ) is highly expressed in tissue from MRS patient and that thalidomide should be considered as an alternative therapy for this uncommon disease.

\section{CASE STUDY}

A 14-year-old boy was seen for swelling of his upper lip, which persisted for approximately 1 year. Initially, the edema was short-lived, periodic, and resolved with antihistamines and corticosteroids. Later, however, labial swelling became permanent. The boy associated the onset of symptoms with the use orthodontic braces and the exacerbations of the lip edema to the ingestion of ketchup. He also reported an upper lip paresthesia and a weight gain of $4 \mathrm{~kg}$ in the previous 3 months. His past medical and family histories were negative for allergies or other relevant pathologies. Physical examination revealed indurated edema, without tenderness in his upper lip, a fissured tongue, and gingival papillae hypertrophy (Fig 1). The patient also had acne on his chin, and metal braces with rubber bands on his teeth.

The laboratory evaluation included a complete blood count, erythrocyte sedimentary rate, $\mathrm{C} 3, \mathrm{C} 4, \mathrm{C} 1$ esterase inhibitor, urine analysis, serum albumin and calcium, whole blood folate, antinuclear antibodies, hepatitis B and C antibodies, total immunoglobulin ( $\mathrm{Ig}) \mathrm{E}$ and latex-specific IgE. Except for a mild elevation of the erythrocyte sedimentary rate, all other results were normal or negative. Skin prick tests for latex, common inhalants, and foods were negative. The patch test, tuberculin intradermal test, and oral challenge with tartrazine and sodium benzoate, controlled by a placebo, were also negative. Chest and face $\mathrm{X}$-ray showed no abnormalities. 


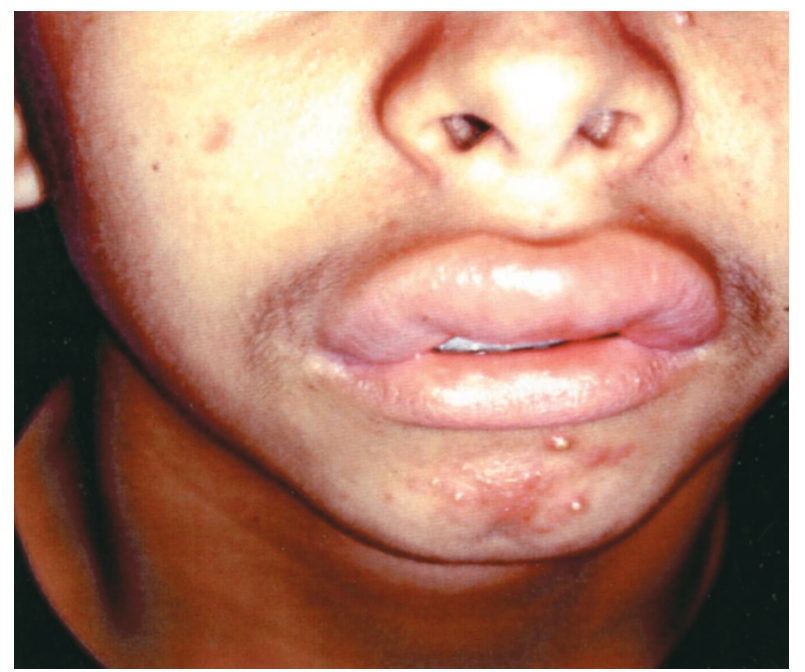

Figure 1. View of lip at first evaluation.

Histologic examination of the involved lip biopsy showed the presence of lymphocytes, macrophages, and epithelioid cells, forming small granulomas in the conjunctive and perivascular areas (Fig 2), compatible with cheilitis granulomatosa. Once the clinical picture of lip edema and histologic examination were available, the diagnosis of MRS was established.

Interested in performing further investigations, we assayed TNF- $\alpha$ in the skin biopsy because it is an important cytokine in chronic granulomatous diseases, such as Crohn disease. ${ }^{18,19} \mathrm{~A}$ high expression of TNF- $\alpha$ was detected in the biopsy sample (Fig 3). The immunohistochemical study was carried out in a 5- $\mu \mathrm{m}$ paraffin-embedded section mounted on poly-L-lysine slides (Sigma Chemical, St. Louis, MO). Endogenous peroxidase was blocked with $3 \% \quad \mathrm{H}_{2} \mathrm{O}_{2}$. Nonspecific binding was blocked with $6 \%$ nonfat milk for 30 minutes at room temperature. After trypsin digestion, sections were stained with rabbit anti-human TNF- $\alpha$, 1:500 (Genzyme Diagnostics, Cambridge, MA) overnight at $4^{\circ} \mathrm{C}$. After incubation with a biotinilated anti-rabbit IgG (1:800 [Dako, Carpinteria, CA]), the slides were developed with streptavidin-peroxidase (1:2,000 [Pearse Chemical, Rockford, IL]) and 3,3-diaminobenzidine tetrahydrochloride (Dako). As a negative control, the primary antibody was replaced by rabbit nonimmune serum and processed in parallel. Sections were counterstained with Gill hematoxylin.

Treatment was begun with $100 \mathrm{mg}$ of thalidomide per day, starting in August 1998. After a 90-day treatment, a reduction in the lip swelling was observed, and thalidomide was maintained for another 60 days, and then stopped. During treatment no adverse side effects were observed. In February 1999, the patient developed a sudden and progressive facial palsy, with a slight deviation of the lip line to the right and left palpebral ptosis, in addition to a slight lip swelling. Remission occurred after a 15-day treatment with dexamethasone, but a relapse occurred after discontinuing treatment, and corticosteroids were reinstated along with thalidomide (100 mg/day). A new remission was obtained and after 30 days, dexamethasone therapy was discontinued with thalidomide remaining (100 mg/day), until March 2000. During this time no new episodes of facial palsy occurred and there was evidence of reduction in lip swelling (Fig 4). His chin acne also cleared up at this time.

\section{DISCUSSION}

Concentrations of proinflammatory cytokines are increased in the intestinal mucosa of patients with active Crohn disease, another granulomatous disease similar to MRS, ${ }^{18}$ and the hypothesis that TNF- $\alpha$ is involved in the pathogenesis of this disease has been supported by the success of therapeutic strategies to antagonize this molecule in Crohn disease. ${ }^{19}$ Likewise, it has been proposed that MRS and Crohn disease could be manifestations of the same disease. ${ }^{20}$ Consequently, it is reasonable to suppose that TNF- $\alpha$ may be involved in the pathogenesis of MRS, based on histologic analyses of these lesions, which show the presence of a granulomatous, noncaseating inflammation. This assumption is supported by our findings of TNF- $\alpha$ in the biopsy sample.

We chose thalidomide to treat this patient because it is a powerful TNF- $\alpha$ inhibitor. ${ }^{21}$ Considering that azathioprine and cyclosporin A act more predominantly at lymphocyte level, one

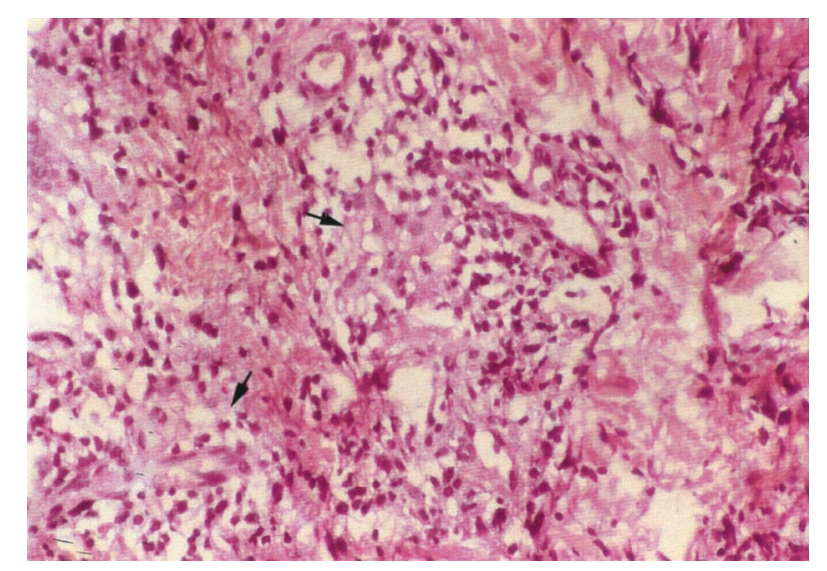

Figure 2. Small granulomas seen in the skin biopsy $(100 \times)$ 


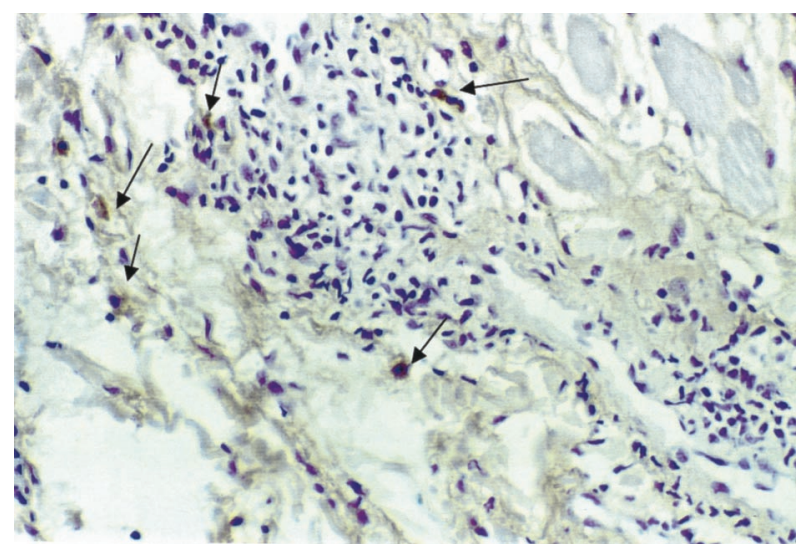

Figure 3. Immunohistochemical assay for TNF- $\alpha$. Positive TNF- $\alpha$ cells (stained brown) are seen around the granuloma (arrows) $400 \times$.

could expect that these drugs would not be the best choice to suppress TNF- $\alpha$ levels. Steroids are potent inhibitors of the inflammatory process, and there is evidence that patients with MRS improve during steroid therapy, although they may relapse after interruption of the drug, as occurred in our patient. It is possible that, similar to other inflammatory diseases, MRS represents a spectrum where some patients could have difficulty controlling the inflammatory process, whereas others may obtain remission of the disease, as occurred in our case report.

Thalidomide is contraindicated in women of childbearing age, and may be associated with side effects such as drowsiness, intestinal constipation, dry- ness of mouth, headache, and rarely, irreversible peripheral neuropathy.

Despite the documentation of TNF- $\alpha$ presence in the lip before the treatment, we were unable to obtain the posttreatment biopsy sample to compare, because the patient refused. However, based on our findings and the patient clinical response, we could speculate that, in this case, thalidomide would act through the modulation of the inflammatory granulomatous process by reducing $\mathrm{TNF}-\alpha$ production at the lesion site.

\section{CONCLUSION}

MRS has to be considered a diagnostic possibility in patients with recurrent orofacial edema associated with neuro-

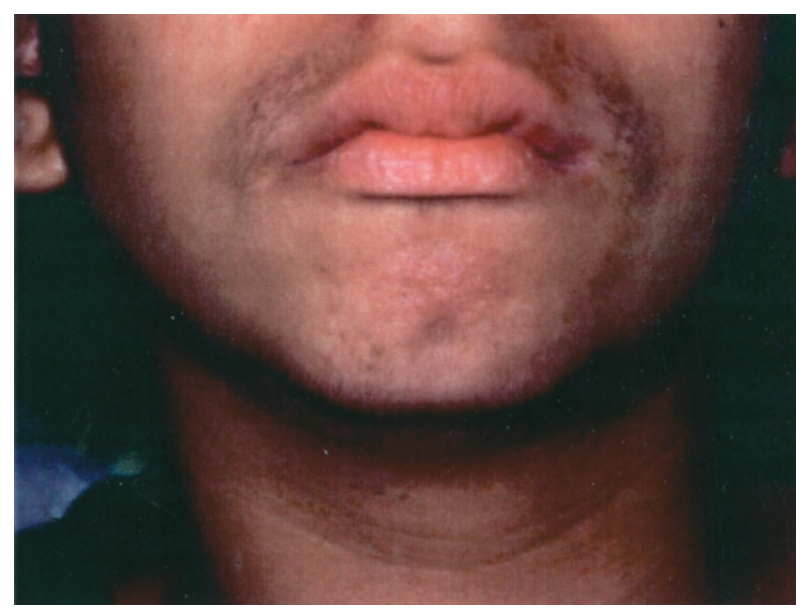

Figure 4. View of lip after 6 months of therapy with thalidomide.

logic or dermatologic manifestations, although not necessarily with the complete triad of symptoms. Additionally, TNF- $\alpha$ might be the main cytokine involved in this disease and, consequently, thalidomide may be considered a therapeutic alternative in these cases, especially in men.

\section{ACKNOWLEDGMENTS}

We thank Bradford S. Hoppe, MD for suggestions and review of the text, and Cristiane Britto, MD for the lip biopsy.

\section{REFERENCES}

1. Rogers RS. Melkersson-Rosenthal syndrome and orofacial granulomatosis. Dermatol Clin 1996;14:371-379.

2. Wiesenfeld D, Ferguson MM, Mitchell $\mathrm{DN}$, et al. Orofacial granulomatosis: a clinical and pathological analysis. Q J Med 1985;54:101-113.

3. Moreno MV, Fernandez M, Oelhing A. Melkersson-Rosenthal syndrome. Allergol Immunopathol 1988;16: 369-372.

4. Worsaae N, Pindborg JJ. Granulomatous gingival manifestations of Melkersson-Rosenthal syndrome. Oral Surg Oral Med Oral Pathol 1980;49: 131-138.

5. Meisel-Stosiek M, Hornstein OP, Stosiek N. Family study on Melkersson-Rosenthal syndrome. Some hereditary aspects of the disease and review of literature. Acta Derm Venereol 1990;70:221-226.

6. Pachor ML, Urbani G, Cortina P, et al. Is the Melkersson-Rosenthal syndrome related to the exposure to food additives? A case report. Oral Surg Oral Med Oral Pathol 1989;67:393-395.

7. Armstrong DK, Biagioni P, Lamey PJ, Burrows D. Contact hypersensitivity in patients with orofacial granulomatosis. Am J Contact Dermat 1997;8:35-38.

8. Levy FS, Bircher AJ, Buchner AS. Delayed-type hypersensitivity to cow's milk protein in Melkersson-Rosenthal syndrome: coincidence or pathogenic role? Dermatology 1996;192:99-102.

9. Pryce DW, King CM. Orofacial granulomatosis associated with delayed hypersensitivity to cobalt. Clin Exp Dermatol 1990;15:384-386.

10. Allen CM, Camisa C, Hamzeh S, Stephens L. Cheilitis granulomatosa: report of six cases and review of the literature. J Am Acad Dermatol 1990; 23:444-450. 
11. Leicht S, Youngberg G, Modica L. Melkersson-Rosenthal syndrome: elevations in serum angiotensin converting enzyme and results of treatment with methotrexate. South Med J 1989; 82:74-76.

12. Kolokotronis A, Antoniades D, Trigonidis G, Papanagiotou P. Granulomatous cheilitis: a study of six cases. Oral Dis 1997;3:188-192.

13. Podmore P, Burrows D. Clofazimine: an effective treatment for MelkerssonRosenthal syndrome or Miescher's cheilitis. Clin Exp Dermatol 1986;11: 173-178.

14. Habel G, O'Regan B. Surgical management of macrocheilia of the lower lip. Br J Oral Maxillofac Surg 1990; 28:295-298.
15. Miralles J, Barnadas MA, de Moragas JM. Cheilitis granulomatosa treated with metronidazole. Dermatology 1995;191:252-253.

16. Olivier V, Lacour JP, Castanet J, et al. Cheilitis granulomatosa in a child. Arch Ped 2000;3:274-277.

17. Stein SL, Mancini AJ. MelkerssonRosenthal syndrome in childhood: successful management with combination steroid and minocycline therapy. J Am Acad Dermatol 1999;41:746-748.

18. Schreiber S, Nikolaus S, Hampe J, et al. Tumour necrosis factor $\alpha$ and interleukin $1 \beta$ in relapse of Crohn's disease. Lancet 1999;353:459-461.

19. Stack WA, Mann SD, Roy AJ. Randomised controlled trial of CDP571 antibody to tumour necrosis factor- $\alpha$ in Crohn's disease. Lancet 1997;349: 521-524.

20. Dummer W, Lurz C, Jeschke R, et al. Granulomatous cheilitis and Crohn's disease in a 3-year-old boy. Pediatr Dermatol 1999;16:39-42.

21. Eigler A, Sinha B, Hartmann G, Endres S. Taming TNF: strategies to restrain this proinflammatory cytokine. Immunol Today 1997;10:487-492.

Requests for reprints should be addressed to: Manoel Medeiros Jr, MD

Av. ACM, 771/1103-Itaigara 41820.000 Salvador, Bahia, Brazil

E-mail: alergo@zaz.com.br 